\title{
On the Implication of a Certain Rate of Convergence to Normality
}

\author{
C. C. HEYDE
}

\section{Introduction}

Consider a sequence of independent and identically distributed random variables $X_{i}, i=1,2,3, \ldots$ which belong to the domain of attraction of the normal distribution. Since this last property implies $E\left|X_{i}\right|<\infty$, we shall suppose for convenience that $E X_{i}=0$. Write

and

$$
S_{n}=\sum_{i=1}^{n} X_{i}, \quad n \geqq 1,
$$

$$
\Phi(x)=\frac{1}{\sqrt{2 \pi}} \int_{-\infty}^{x} e^{-\frac{1}{2} u^{2}} d u .
$$

We shall establish the following main theorem.

Theorem 1. If there is a monotone sequence of normalizing constants $\left\{B_{n}\right\}$ with $B_{n} \rightarrow \infty$ as $n \rightarrow \infty$ such that

then $E X_{i}^{2}<\infty$.

$$
\sum_{n=3}^{\infty} n^{-1}(\log \log n) \sup _{x}\left|\operatorname{Pr}\left(S_{n} \leqq B_{n} x\right)-\Phi(x)\right|<\infty,
$$

This result extends that contained in Theorem 3.1 of Ibragimov [7] where it is shown that if

$$
\sup _{x}\left|\operatorname{Pr}\left(S_{n} \leqq B_{n} x\right)-\Phi(x)\right|=0\left(n^{-\delta}\right)
$$

for some $\delta>0$, then $E X_{i}^{2}<\infty$. It provides us with a converse to a result of Heyde [4]. Furthermore, it provides an interesting elucidation of a result of Petrov [8] concerning iterated logarithm behaviour which was proved without explicit assumptions regarding the existence of moments. Theorem 1 is proved in Section 2 while Section 3 contains a discussion of its applications.

\section{Proof of Theorem 1}

Suppose firstly that the $X_{i}$ are symmetric. Since

as $x \rightarrow \infty$, we have

$$
1-\Phi(x) \sim \frac{1}{x \sqrt{2 \pi}} e^{-\frac{1}{2} x^{2}}
$$

$$
\sum_{n=3}^{\infty} n^{-1}(\log \log n)\left\{1-\Phi\left[((2+\varepsilon) \log \log n)^{\frac{1}{2}}\right]\right\}<\infty
$$


for any $\varepsilon>0$ so that, writing $t_{n}=[(2+\varepsilon) \log \log n]^{\frac{1}{2}}$, it follows from (1) that

$$
\sum_{n=3}^{\infty} n^{-1}(\log \log n) \operatorname{Pr}\left(\left|S_{n}\right|>B_{n} t_{n}\right)<\infty .
$$

But, for symmetric random variables,

$$
\operatorname{Pr}\left(\left|S_{n}\right|>B_{n} t_{n}\right) \geqq \frac{1}{2} \operatorname{Pr}\left(\max _{1 \leqq k \leqq n}\left|X_{k}\right|>B_{n} t_{n}\right),
$$

(e.g. Feller [2], II, 147) and, from Bonferroni's inequalities (e.g. [2], I, 100) we have

so that

$$
\begin{aligned}
n \operatorname{Pr}\left(\left|X_{i}\right|>B_{n} t_{n}\right)\left[1-\frac{1}{2}(n-1) \operatorname{Pr}\left(\left|X_{i}\right|>B_{n} t_{n}\right)\right] \\
\quad \leqq \operatorname{Pr}\left(\max _{1 \leqq k \leqq n}\left|X_{k}\right|>B_{n} t_{n}\right) \leqq n \operatorname{Pr}\left(\left|X_{i}\right|>B_{n} t_{n}\right),
\end{aligned}
$$

$$
\operatorname{Pr}\left(\max _{1 \leqq k \leqq n}\left|X_{k}\right|>B_{n} t_{n}\right) \sim n \operatorname{Pr}\left(\left|X_{i}\right|>B_{n} t_{n}\right)
$$

as $n \rightarrow \infty$ and hence, from (2), (3) and (4),

$$
\sum_{n=3}^{\infty}(\log \log n) \operatorname{Pr}\left(\left|X_{i}\right|>B_{n} t_{n}\right)<\infty .
$$

Now suppose that $E X_{i}^{2}=\infty$ so that

$$
K(t)=\int_{|x t| \leqq 1} x^{2} d \operatorname{Pr}\left(X_{i} \leqq x\right) \rightarrow \infty
$$

as $t \rightarrow 0$. Since the $X_{i}$ belong to the domain of attraction of the normal distribution, $K(t)$ is slowly varying as $t \rightarrow 0$ (e.g. [2], II, 303) and $n B_{n}^{-2} K\left(B_{n}^{-1}\right) \rightarrow 1$ as $n \rightarrow \infty$ (e.g. [2], II, 304-305).

Then, we have

$$
\begin{aligned}
\int_{|x| \leqq B_{n} t_{n}} x^{2} d \operatorname{Pr}\left(X_{i} \leqq x\right) \leqq & \int_{|x| \leqq B_{3} t_{3}} x^{2} d \operatorname{Pr}\left(X_{i} \leqq x\right) \\
& +\sum_{k=3}^{n} B_{k+1}^{2} t_{k+1}^{2} \operatorname{Pr}\left(B_{k} t_{k}<\left|X_{i}\right| \leqq B_{k+1} t_{k+1}\right) \\
\leqq & \int_{|x| \leqq B_{3} t_{3}} x^{2} d \operatorname{Pr}\left(X_{i} \leqq x\right) \\
& +C \sum_{k=3}^{n} K\left(B_{k}^{-1}\right) k \log \log k \operatorname{Pr}\left(B_{k} t_{k}<\left|X_{i}\right| \leqq B_{k+1} t_{k+1}\right)
\end{aligned}
$$

for some positive constant $C$. Furthermore, it is easily shown, using (5), that

$$
\sum_{k=3}^{\infty} k(\log \log k) \operatorname{Pr}\left(B_{k} t_{k}<\left|X_{i}\right| \leqq B_{k+1} t_{k+1}\right)<\infty .
$$

Thus, using (6), (7) and by virtue of our assumption that $K(t) \rightarrow \infty$ as $t \rightarrow 0$, we have from the Kronecker lemma that

$$
n B_{n}^{-2} \int_{|x| \leqq B_{n} t_{n}} x^{2} d \operatorname{Pr}\left(X_{i} \leqq x\right) \sim\left[K\left(B_{n}^{-1}\right)\right]^{-1} K\left(B_{n}^{-1} t_{n}^{-1}\right) \rightarrow 0
$$


as $n \rightarrow \infty$. This establishes the required contradiction since by definition,

$$
\left[K\left(B_{n}^{-1}\right)\right]^{-1} K\left(B_{n}^{-1} t_{n}^{-1}\right) \geqq 1 .
$$

It follows that (5) is only possible in the case where $K\left(B_{n}^{-1}\right)$ approaches a positive constant as $n \rightarrow \infty$ in which case it is easily seen that $E X_{i}^{2}<\infty$. This completes the proof for the case of symmetric $X_{i}$.

In order to deal with the general case, consider the sequence $Y_{i}, i=1,2,3, \ldots$ of independent, symmetrized random variables; each $Y_{i}$ having the distribution of the difference between two independent $X_{i}^{\prime}$ s. Write

$$
G_{n}(x)=\operatorname{Pr}\left(Y_{1}+\cdots+Y_{n} \leqq x B_{n} \sqrt{2}\right), \quad F_{n}(x)=\operatorname{Pr}\left(S_{n} \leqq B_{n} x\right) .
$$

Then, using $*$ to denote convolution,

$$
G_{n}(x)=F_{n}(x \sqrt{2}) *\left(1-F_{n}(-x \sqrt{2}-0)\right),
$$

and in view of (1) we obtain

$$
\begin{aligned}
& \sum_{n=3}^{\infty} n^{-1}(\log \log n) \sup _{x}\left|G_{n}(x)-\Phi(x)\right| \\
&=\sum_{n=3}^{\infty} n^{-1}(\log \log n) \sup _{x} \mid F_{n}(x \sqrt{2}) *\left(1-F_{n}(-x \sqrt{2}-0)\right) \\
&-\Phi(x \sqrt{2}) *(1-\Phi(-x \sqrt{2})) \mid \\
& \leqq \sum_{n=3}^{\infty} n^{-1}(\log \log n) \sup _{x} \mid F_{n}(x \sqrt{2}) *\left(1-F_{n}(-x \sqrt{2}-0)\right) \\
&-\Phi(x \sqrt{2}) *\left(1-F_{n}(-x \sqrt{2}-0)\right) \mid \\
&+\sum_{n=3}^{\infty} n^{-1}(\log \log n) \sup _{x} \mid \Phi(x \sqrt{2}) *\left(1-F_{n}(-x \sqrt{2}-0)\right) \\
&-\Phi(x \sqrt{2}) *(1-\Phi(-x \sqrt{2})) \mid<\infty
\end{aligned}
$$

Thus, our result for the symmetric case gives $E Y_{i}^{2}<\infty$ which implies $E X_{i}^{2}<\infty$. This completes the proof of the theorem.

Remark 1. The following result of Davis [1] is worth noting in connection with Theorem 1. If $E X_{i}=0, E X_{i}^{2}=1, E X_{i}^{2} \log ^{+} \log ^{+}\left|X_{i}\right|<\infty$ and

then

$$
\beta_{n}^{2}=\int_{|x|<\sqrt{n}} x^{2} d \operatorname{Pr}\left(X_{i} \leqq x\right)-\left(\int_{|x|<\sqrt{n}} x d \operatorname{Pr}\left(X_{i} \leqq x\right)\right)^{2},
$$

$$
\sum_{n=3}^{\infty} n^{-1}(\log \log n) \sup _{x}\left|\operatorname{Pr}\left(S_{n} \leqq x \beta_{n} \sqrt{n}\right)-\Phi(x)\right|<\infty .
$$

$\left(\right.$ Here $\log ^{+} x$ denotes $\max (0, \log x)$.) 
Remark 2. It is conjectured that an improved version of Theorem 1 will hold with the $\log \log n$ term omitted in (1). For background to this conjecture see Heyde [5].

\section{Applications of Theorem 1}

Firstly we use Theorem 1 to elucidate a result of Heyde [4].

Theorem 2. Take $0<\delta<1$. Let $E\left|X_{i}\right|^{2+\delta}<\infty$ and write $B_{n}^{2}=n E X_{i}^{2}\left(1+\varepsilon_{n}\right)$. Then, if $\sum_{1}^{\infty} n^{-1+\delta / 2}\left|\varepsilon_{n}\right|<\infty$, we obtain

$$
\sum_{n=1}^{\infty} n^{-1+\delta / 2} \sup _{x}\left|\operatorname{Pr}\left(S_{n} \leqq B_{n} x\right)-\Phi(x)\right|<\infty .
$$

Conversely, if (8) holds for some monotone sequence $\left\{B_{n}\right\}$, and the $X_{i}$ belong to the domain of attraction of the normal distribution, then $E X_{i}^{2}<\infty$ and $B_{n}^{2}=n E X_{i}^{2}\left(1+\varepsilon_{n}\right)$ where $\varepsilon_{n} \rightarrow 0$ as $n \rightarrow \infty$. If, in addition, $\sum_{1}^{\infty} n^{-1+\delta / 2}\left|\varepsilon_{n}\right|<\infty$, then $E\left|X_{i}\right|^{2+\delta}<\infty$.

Proof. Write $E X_{i}^{2}=\sigma^{2}(<\infty)$. If $E\left|X_{i}\right|^{2+\delta}<\infty$, we obtain from the theorem of $[4]$ that

$$
\sum_{n=1}^{\infty} n^{-1+\delta / 2} \sup _{x}\left|\operatorname{Pr}\left(S_{n} \leqq x \sigma \sqrt{n}\right)-\Phi(x)\right|<\infty
$$

Furthermore,

$$
\begin{aligned}
\operatorname{Pr}\left(S_{n}\right. & \left.\left.\leqq x \sigma \sqrt{n\left(1+\varepsilon_{n}\right.}\right)\right)-\Phi(x) \\
& =\operatorname{Pr}\left(S_{n} \leqq\left(x \sqrt{1+\varepsilon_{n}} \sigma \sqrt{n}\right)-\Phi\left(x \sqrt{1+\varepsilon_{n}}\right)+\Phi\left(x \sqrt{1+\varepsilon_{n}}\right)-\Phi(x),\right.
\end{aligned}
$$

while expanding by Taylor's theorem gives

$$
\Phi\left(x \sqrt{1+\varepsilon_{n}}\right)-\Phi(x)=\frac{1}{2 \sqrt{2 \pi}} \varepsilon_{n} x e^{-\frac{1}{2} x^{2}}+0\left(\varepsilon_{n}^{2}\right) .
$$

Thus, upon taking sup with respect to $x$ in (10) and using (11), we obtain

$$
\left.\sup _{x} \mid \operatorname{Pr}\left(S_{n} \leqq x \sigma \sqrt{n\left(1+\varepsilon_{n}\right.}\right)\right)-\Phi(x)\left|\leqq \sup _{x}\right| \operatorname{Pr}\left(S_{n} \leqq x \sigma \sqrt{n}\right)-\Phi(x)|+C| \varepsilon_{n} \mid,
$$

for some positive constant $C$. The result of the first part of the theorem then follows from (9) and (12).

Now suppose that (8) holds for some monotone sequence $\left\{B_{n}\right\}$, and the $X_{i}$ belong to the domain of attraction of the normal distribution. It follows from Theorem 1 that $E X_{i}^{2}=\sigma^{2}<\infty$, and consequently $B_{n}$ must be of the form $\left.B_{n}=\sigma \sqrt{n\left(1+\varepsilon_{n}\right.}\right)$ where $\varepsilon_{n} \rightarrow 0$ as $n \rightarrow \infty$. Further, the equation (10) can be rewritten to yield

$$
\left.\sup _{x}\left|\operatorname{Pr}\left(S_{n} \leqq x \sigma \sqrt{n}\right)-\Phi(x)\right| \leqq \sup _{x} \mid \operatorname{Pr}\left(S_{n} \leqq x \sigma \sqrt{n\left(1+\varepsilon_{n}\right.}\right)\right)-\Phi(x)\left|+C^{\prime}\right| \varepsilon_{n} \mid,
$$


where $C^{\prime}$ is a positive constant, so that, if $\sum_{1}^{\infty} n^{-1+\delta / 2}\left|\varepsilon_{n}\right|<\infty$, (9) holds. The result $E\left|X_{i}\right|^{2+\delta}<\infty$ then follows from the theorem of [4]. This completes the proof.

Our next application of Theorem 1 concerns its relation to questions of behaviour of iterated logarithm type. It is well-known that

$$
\operatorname{Pr}\left(\limsup _{n \rightarrow \infty}(2 n \log \log n)^{-\frac{1}{2}} S_{n}=1\right)=1
$$

if and only if $E X_{i}=0, E X_{i}^{2}=1$ (Hartman and Wintner [3] obtained the sufficiency part and Strassen [9] the necessity). However, it has been shown by Freedman (see [9]) that there exist symmetric random variables $X_{i}$ with $E X_{i}^{2}=\infty$ or even $E\left|X_{i}\right|=\infty$ for which it is possible to choose a monotone sequence $\left\{b_{n}\right\}$ of positive constants with $b_{n} \rightarrow \infty$ as $n \rightarrow \infty$ such that

$$
\operatorname{Pr}\left(\limsup _{n \rightarrow \infty} b_{n}^{-1} S_{n}=1\right)=1 .
$$

Furthermore, Heyde [6] has shown that the behaviour (13) is only possible if the $X_{i}$ belong to the domain of partial attraction of the normal distribution. Also, the construction of Freedman's is quite inexplicit and it is possible to obtain very little idea of the generality of the circumstances under which behaviour of the type (13) is possible when $E X_{i}^{2}=\infty$.

Now the following theorem has recently been obtained by Petrov [8].

Theorem. Suppose that the independent random variables $X_{i}, i=1,2,3, \ldots$ have symmetric distributions. If there exists a sequence of constants $\left\{C_{n}\right\}$ such that

and

$$
C_{n} \uparrow \infty, \quad C_{n}^{-1} C_{n+1} \rightarrow 1
$$

$$
\sup _{x}\left|\operatorname{Pr}\left(\sum_{i=1}^{n} X_{i}<C_{n}^{\frac{1}{2}} x\right)-\Phi(x)\right|=0\left(\left(\log C_{n}\right)^{-1-\delta}\right)
$$

as $n \rightarrow \infty$ for some $\delta>0$, then

$$
\operatorname{Pr}\left(\limsup _{n \rightarrow \infty}\left(2 C_{n} \log \log C_{n}\right)^{-\frac{1}{2}} \sum_{i=1}^{n} X_{i}=1\right)=1 .
$$

This theorem involves no explicit assumptions regarding the existence of moments and it is an interesting question as to whether it can be used to generate results of the type of (13) for identically distributed random variables with $E X_{i}^{2}=\infty$. The answer to this question is in the negative. This follows immediately from Theorem 1 upon noting that, in the case of identically distributed $X_{i}$,

as $n \rightarrow \infty$.

$$
\log C_{n} \sim \frac{1}{2} \log n
$$

\section{References}

1. Davis, J.A.: Convergence rates for the law of the iterated logarithm. Ann. math. Statistics 39, 1479-1485 (1968).

2. Feller, W.: An introduction to probability theory and its applications, vols. I, II. New York: Wiley $1957,1966$.

3. Hartman, P., Wintner, A.: On the law of the iterated logarithm. Amer. J. Math. 63, 169-176 (1941). 
4. Heyde, C. C.: On the influence of moments on the rate of convergence to the normal distribution. Z. Wahrscheinlichkeitstheorie verw. Geb. 8, 12-18 (1967).

5. - Some properties of metrics in a study on convergence to normality. Z. Wahrscheinlichkeitstheorie verw. Geb. 11, 181-192 (1969).

6. - A note concerning behaviour of iterated logarithm type. Proc. Amer. math. Soc. (1969) (in press).

7. Ibragimov, I. A.: On the accuracy of the Gaussian approximation to the distribution function of sums of independent variables. Theor. Probab. Appl. 11, 559-576 (1966).

8. Petrov, V.V.: On the law of the iterated logarithm without assumptions about the existence of moments. Proc. Nat. Acad. Sci. U.S.A. 59, 1068-1072 (1968).

9. Strassen, V.: A converse to the law of the iterated logarithm. Z. Wahrscheinlichkeitstheorie verw. Geb. 4, 265-268 (1966).

\author{
Dr. C. C. Heyde \\ Department of Statistics \\ Australian National University \\ School of General Studies \\ Box 4, P.O. \\ Canberra, A.C.T. 2600 \\ Australia
}

(Received August 18, 1969) 